\title{
Effects of Bufei Yishen formula on klotho gene expression and inflammation-related RIG-I/NF-kB pathway in cigarette smoke extract-treated BEAS-2B cells
}

\author{
Wei-min Wang ${ }^{1,2}$, Long-chuan $\mathrm{Wu}^{2}$, Bo Chen ${ }^{1}$, Yan $\mathrm{Li}^{1}, \mathrm{Yu}$ Chen ${ }^{2}$, Xing-xing $\mathrm{Xu}{ }^{2}$, \\ Jian-qing $\mathbf{W u}^{1}$ \\ ${ }^{1}$ Department of Geriatrics, The First Affiliated Hospital of Nanjing Medical University, Nanjing, ${ }^{2}$ Department of Respiratory \\ Medicine, Huaian Hospital of Traditional Chinese Medicine, Huaian, Jiangsu, China
}

*For correspondence: Email: Ik1262@163.com

\begin{abstract}
Purpose: To investigate the anti-inflammatory effect of Bufei yishen formula on chronic obstructive pulmonary disease (COPD) and klotho expression in cigarette smoke extract (CSE)-treated BEAS-2B cells.

Methods: Cigarette smoke extract (CSE) was diluted in Dulbecco's modified Eagle's medium (DMEM) and used to treat a COPD cell model established using BEAS-2B cells. The CSE-treated cells were transfected with klotho overexpression plasmid, and the COPD cell model was treated with Bufei yishen formula. Dexamethasone was used as positive control. Changes in the expressions of klotho, RIG-I, and NF-KB were determined using reverse transcription-polymerase chain reaction (RT-PCR) and Western blotting, while enzyme linked immunosorbent assay (ELISA) was used to evaluate changes in the expressions of IL- 6 and TNF- $\alpha$.

Results: Klotho expression level was reduced in COPD model. Overexpression of klotho inhibited the expressions of IL-6, TNF- $\alpha$, RIG-I, NF-KB, but bufei yishen formula increased klotho expression level and decreased the expressions of RIG-I, NF-KB, IL-6 and TNF- $\alpha$. However, after CSE treatment, the anti-inflammatory effect of the formula decreased significantly when klotho expression was knocked down.

Conclusion: Bufei yishen formula possesses anti-inflammatory effects which are significantly decreased after klotho knockdown. This suggests that the formula probably acts by upregulating klotho expression, thereby down-regulating the expressions of IL-6 and TNF- $\alpha$ via RIG-I/NF-KB pathway.
\end{abstract}

Keywords: Bufei yishen formula, COPD, Klotho, Inflammation, RIG-I/NF-KB

\begin{abstract}
This is an Open Access article that uses a funding model which does not charge readers or their institutions for access and distributed under the terms of the Creative Commons Attribution License (http://creativecommons.org/licenses/by/4.0) and the Budapest Open Access Initiative (http://www.budapestopenaccessinitiative.org/read), which permit unrestricted use, distribution, and reproduction in any medium, provided the original work is properly credited.

Tropical Journal of Pharmaceutical Research is indexed by Science Citation Index (SciSearch), Scopus, International Pharmaceutical Abstract, Chemical Abstracts, Embase, Index Copernicus, EBSCO, African Index Medicus, JournalSeek, Journal Citation Reports/Science Edition, Directory of Open Access Journals (DOAJ), African Journal Online, Bioline International, Open-J-Gate and Pharmacy Abstracts
\end{abstract}

\section{INTRODUCTION}

Chronic obstructive pulmonary disease (COPD), characterized by persistent poor airflow, is preventable and curable. The limited airflow is related to inflammatory responses in airway and lung tissues to pernicious smoke or cigarette particles [1]. Chronic exposure to smoke can induce oxidative stress responses in lung and liver, resulting in cell aging $[2,3]$. 
Klotho is an anti-aging protein which has been reported to have anti-inflammatory effects $[4,5]$. Studies have shown that klotho protects against inflammation and aging by downregulating the expression of retinoic acid-inducible gene-I (RIGI) or nuclear factor $\mathrm{kB}(\mathrm{NF}-\mathrm{kB})$, and also by suppressing the release of interleukin-6 (IL-6) [6]. It can be hypothesized that klotho prevents inflammation by downregulating IL-6 expression via RIG-I/NF-kB pathway in COPD.

Current treatments for COPD focus mainly on symptom alleviation and resistance to inflammation: they do not prevent lung aging. According to the theory of Chinese traditional medicine, patients with mild and serious COPD generally have $Q i$ deficiency in pulmonary and renal tissues, which are represented by metal and water, respectively. Regulating and nourishing the lung and kidney can improve asthma, mobility, and immunity and reduce the risk of serious events. This represents the unique concept of holistic therapy in Chinese medicine.

According to previous research [7], Bufei yishen formula is safe and efficient in patients with stable COPD, and it can alleviate symptoms and improve lung function. The formula contains six herbs: astragalus, ginseng, rehmannia, cornus, epimedium and atractylodes. These herbs are used for anti-inflammation and anti-aging. Klotho protein prevents aging and suppresses COPDinflammation. Thus, it was hypothesized that the formula prevents inflammation and cures COPD probably by regulating klotho expression through RIG-I/NF-kB. Therefore, a lung epithelial cell line infected with klotho-targeting small interfering RNA (siRNA) and a COPD cell model treated with CSE were used to determine if bufei yishen formula exerts anti-inflammatory and anti-aging effects by regulating klotho expression through the RIG-I/NF-KB pathway.

\section{EXPERIMENTAL}

\section{Cell culture and CSE treatment}

BEAS-2B cells (human lung epithelial cell line) were obtained from American Type Culture Collection (Manassas, VA), and incubated in Dulbecco's modified Eagle's medium (DMEM) with $10 \%$ fetal bovine serum (FBS) and antibiotics at $37{ }^{\circ} \mathrm{C}$ in an atmosphere containing $5 \% \mathrm{CO}_{2}$. Cigarette smoke extract (CSE, 40 $\mathrm{mg} / \mathrm{mL}$ in DMSO) containing $6 \%$ nicotine at -80 ${ }^{\circ} \mathrm{C}$, was purchased from Murty Pharmaceuticals (Lexington, $\mathrm{KY}$ ), and was mixed with cell medium before use. This research was approved by the Ethical Committee of Department of Geriatrics, The First Affiliated Hospital of Nanjing Medical
University, Nanjing, Jiangsu, China (approval no. 20186114), and was carried out according to the guidelines of Declaration of Helsinki promulgated in 1964, as amended in 1996 [8].

\section{Preparation of overexpressed klotho}

BEAS-2B cells were incubated in $10 \%$ FBS without antibiotics and transfected with $2500 \mathrm{ng}$ lentiviral vectors carrying the complete open reading frame (ORF) of the human klotho gene (including the transmembrane domain) on the second day. A second group of BEAS-2B cells was transfected with lentiviral vector without the ORF of the human klotho gene. The cells were incubated in fresh medium with $\mathrm{G} 418(1 \mathrm{mg} / \mathrm{mL}$, diluted 20 times) after transfection for $24 \mathrm{~h}$. Fourteen days after the addition of G418, an individual clone (P1) was selected. The expression levels of all clones were measured by RT-qPCR. Klotho expression level in the cells transfected with empty vector was similar to that of normal BEAS-2B cells which were used as blank control cells. Cells transfected with the ORF of the klotho gene which showed the highest klotho expression level were used as klotho-overexpressing cells. Sub-cultured cells (P2-P13) were used in all experiments.

\section{Klotho siRNA transfection, qRT-PCR and Western blotting}

Klotho siRNA and control siRNA were transfected into BEAS-2B cells, and the cells were transferred to $10 \%$ FBS-containing medium after 6-h transfection. Protein and RNA were extracted after $48 \mathrm{~h}$ according to the kit protocol. Primers specific for klotho, NF-kB/p65 and RIG-I were designed, and the expressions of their corresponding mRNAs were determined using RT-qPCR according to manufacturer's protocol (Applied Biosystems, USA). Cellular protein extraction was also performed according to the manufacturer's protocol (Applied Biosystems, USA). The expressions of klotho, NF-KB/p65 and RIG-I were assayed in line with Western blotting protocol.

\section{Determination of the expressions of IL- 6 and TNF- $\alpha$}

Samples were collected in line with the cell lysis solution protocol, and the expression levels of IL6 and TNF- $\alpha$ were evaluated according to ELISA protocol of IL-6 and TNF- $\alpha$ (Affymetrix, USA).

\section{Statistical analysis}

Data are presented as mean \pm standard deviation (SD). They were analyzed statistically 
with SPSS version 16.0. Differences between two groups were evaluated using $t$-test. Values of $p<0.05$ were assumed to indicate statistical significance.

\section{RESULTS}

CSE-treated BEAS-2B cells suppressed klotho expression and induced inflammation via RIG-I/NF-KB signaling pathway

Results from RT-qPCR assays showed that mRNA expression levels of RIG-I and NF-kB-P65 in CSE-treated BEAS-2B cells were significantly higher $(p<0.05)$ than those in the control cells (Figure 1). Western blotting showed that protein expression levels of RIG-I and NF-kB-P65 were also significantly increased (Figure $1 \mathrm{C}, \mathrm{D}$ and $\mathrm{E}$; $p<0.05)$. Furthermore, results from ELISA demonstrated that the expressions of the inflammatory biomarkers IL- 6 and TNF- $\alpha$ were significantly upregulated in response to CSE $(p<$ 0.05).

The klotho gene expression in BEAS-2B cells was inhibited concentration-dependently by CSE.

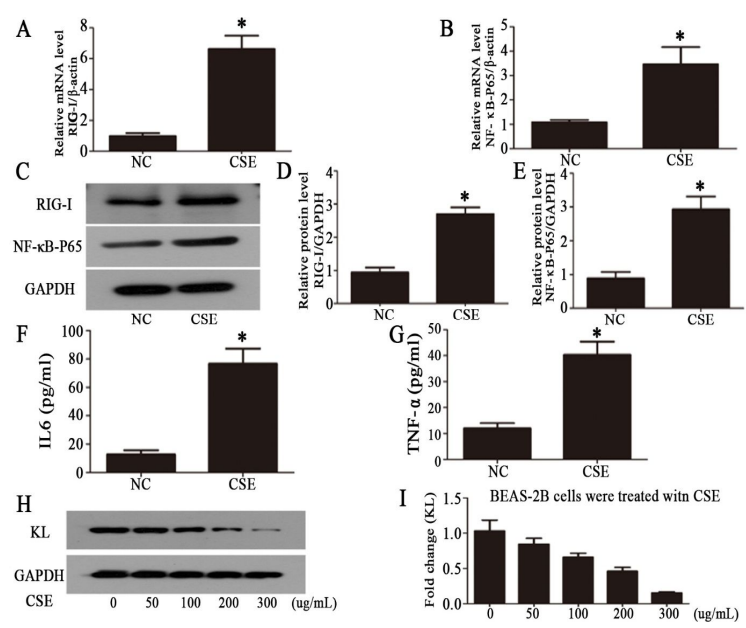

Figure 1: CSE-induced inflammatory responses in BEAS-2B cells. A \& B: mRNA expression of RIG-I and NF-kB-P65 by qPCR after CSE treatment; C, D \& E: Protein expressions of RIG-I and NF-kB-P65 after CSE treatment; $F$ \& $G$ : Expressions of the biomarkers IL-6 and TNF- $\alpha$; H \& I: CSE suppressed klotho protein expression

Overexpression of klotho suppressed CSEinduced inflammation via RIG-I/NF-kB signaling pathway

Results from RT-PCR showed that after CSE treatment, mRNA expression levels of RIG-I and $\mathrm{NF}-\mathrm{kB} / \mathrm{p} 65$ were significantly lower in the klothooverexpression group $(p<0.05)$ than in the blank vector control group (Figure $2 \mathrm{~A}$ and $\mathrm{B}$ ). Consistent with these findings, the protein expression levels of RIG-I and NF-kB/p65 were significantly reduced $(p<0.05)$, but the reductions were milder than those in the positive control group (dexamethasone group) (Figure 2 C, D and E). Furthermore, ELISA showed that the expression levels of the inflammatory biomarkers IL-6 and TNF- $\alpha$ were significantly decreased following klotho overexpression $(p<$ 0.05).
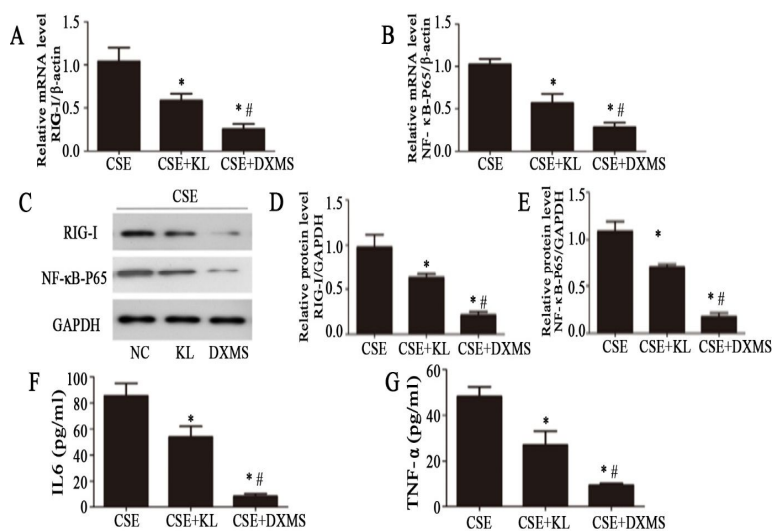

Figure 2: Suppression of CSE-induced inflammation by klotho. A \& B: mRNA expressions of RIG-I and NFKb-P65 after CSE treatment; ${ }^{*} p<0.05$, compared with the normal expression group); \#p<0.05, compared with the overexpression group; C, D \& E: Protein expressions of RIG-I and NF-KB-P65 after CSE treatment; ${ }^{*} p<0.05$, compared with the normal expression group; \#p<0.05, compared with the overexpressed group; F \& G: Overexpressed klotho suppressed the expressions of IL- 6 and TNF- $\alpha$; ${ }^{*} p<$ 0.05 , compared with the normal expression group; \#p $<0.05$, compared with the overexpression group

\section{Bufei yishen formula suppressed inflammation in CSE-treated BEAS-2B cells via RIG-I/NF-KB signaling pathway}

BEAS-2B cells were treated with the bufei yishen formula for $24 \mathrm{~h}$ after CSE treatment, and inflammation-related pathways and biomarkers were determined. Results from RT-qPCR showed that compared with the control group which was treated with CSE only, inflammation in the formula group was significantly alleviated, and mRNA expression levels of RIG-I and NF-KB were significantly decreased $(p<0.05)$. Western blotting results showed that protein expression levels of RIG-I and NF-KB were significantly reduced in the bufei vishen formula group (Figures $3 \mathrm{C}, \mathrm{D}$ and $\mathrm{E} ; p<0.05$ ). ELISA results showed that the expression levels of IL-6 and TNF- $\alpha$ were also significantly reduced (Figure 3 $\mathrm{F}, \mathrm{G} ; p<0.05)$, consistent with previous results. However, the anti-inflammatory effect in the formula group was inferior to that in the positive control group (dexamethasone group). 


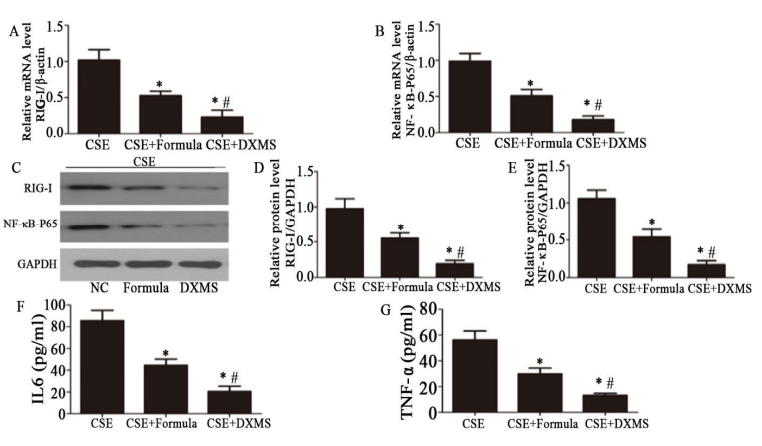

Figure 3: Suppression of CSE-induced inflammation by Bufei yishen formula. A \& B: mRNA expressions of RIG-I and NF-kB-P65 after CSE and formula treatments; ${ }^{*} p<0.05$, compared with the control group; $\# p<0.05$, compared with the formula group; C, D \& E: Protein expressions of RIG-I and NF-KB-P65 after CSE and formula treatments; ${ }^{*} p<0.05$, compared with the control group; $\# p<0.05$, compared with the formula group; $F$ \& G: Suppression of the expressions of IL- 6 and TNF- $\alpha$ by formula-mediated klotho induction; ${ }^{*} p<0.05$, compared with the control group; $\# p<0.05$, compared with the formula group

\section{Bufei yishen formula prevented inflammation by upregulating klotho expression}

The anti-inflammatory effect of bufei yishen formula became weaker with klotho knockdown. Results from RT-qPCR demonstrated that the mRNA expression levels of RIG-I and NF-KB were significantly higher in the klotho knockdown group than in the formula-only group (Figures 4D $\& 4 \mathrm{E}, p<0.05$ ). The protein expressions (Figure $4 \mathrm{~F}, 4 \mathrm{G} \& 4 \mathrm{H}$ ) and ELISA results (Figure $4 \mathrm{I} \& 4 \mathrm{~J}$ ) were consistent with previous results.

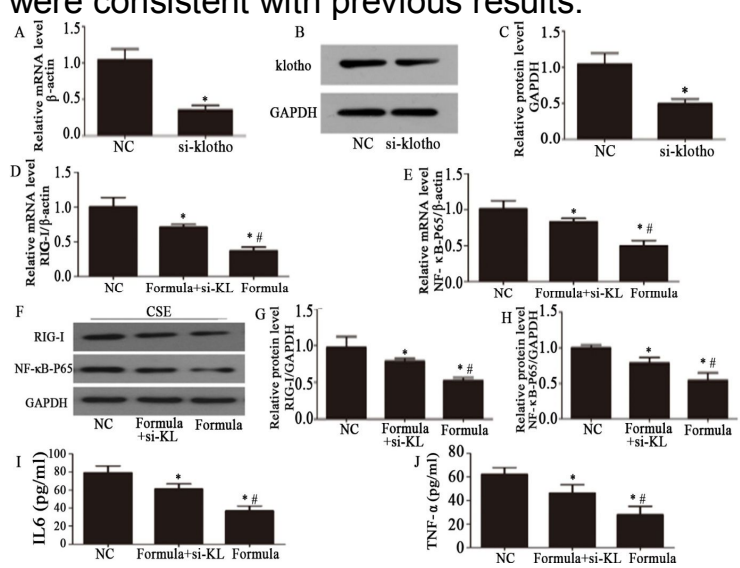

Figure 4: Bufei yishen formula upregulated klotho expression. A: RT-qPCR was used to assess the outcome of siRNA-mediated klotho knockdown; * $p<$ 0.05 , compared with the control group; B \& C: Western blot was used to assess the outcome of siRNAmediated Klotho knockdown; * $p<0.05$; compared with the control group; D \& E: mRNA expressions of RIG-I and NF-KB-P65 after CSE and formula treatments, and klotho knockdown; ${ }^{*} p<0.05$, compared with the control group; $\# p<0.05$, compared with knockeddown klotho group; $F, G$ \& H: Protein expressions of RIG-I and NF-KB-P65 after CSE and formula treatments, and klotho knockdown; ${ }^{*} p<0.05$ compared with the control group; $\# p<0.05$, compared with the klotho knockdown group; I \& J: ELISA results for the expressions of the inflammatory biomarkers IL6 and TNF- $\alpha$ after CSE and formula treatments and klotho knockdown, ${ }^{*} p<0.05$, compared with the control group; $\# p<0.05$, compared with the klotho knockdown group

\section{DISCUSSION}

Chronic obstructive pulmonary disease (COPD) has been identified as a common, preventable and curable condition [1]. Oxidative stress accelerates epithelial cell death and alveolar deconstruction, which lead to pulmonary emphysema, airflow limitation and expiratory dyspnea. Lung aging is related to anatomical and functional changes in physiological aspects such as alveolar expansion, airflow limitation, and delayed expiration. Aging is a natural process, but smoke can stimulate and accelerate the aging of epithelial cells. Thus, elderly individuals who smoke have an increased incidence of respiratory diseases. Chronic obstructive pulmonary disease (COPD) is caused mainly by disturbance in air passage and pulmonary alveoli with typical clinical manifestations of persistent respiratory symptoms and limited airflow. It is currently thought that the disease is related to airway inflammation, imbalance between proteases and protease inhibitors, and oxidative stress; while long-term exposure to smoke and toxic particles can promote the disease. Chronic exposure to smoke induces oxidative stress and cell aging. Therefore, mechanisms underlying COPD pathogenesis such as cell aging, inflammation, oxidative stress and changes in expressions of anti-aging molecules are all involved in regulation of aging.

Gene mapping experiments were initially employed for assessing the relation between inflammation and aging. It is well known that inflammatory factors such as IL- 6 and TNF- $\alpha$ accelerate the aging process $[9,10]$. However, the underlying mechanism is still unclear. Klotho is an anti-aging protein, and previous studies have found that mice lacking klotho display aging phenotypes similar to those of humans, while mice with increased klotho levels are resistant to aging and have longer lifespans. In addition to its anti-aging effects, multiple studies have reported the anti-inflammatory effects of klotho.

It has been reported that klotho suppressed RIGI-mediated IL-6 and IL-8 expressions in a dosedependent manner. The upregulations of RIG-I, IL-6 and IL-8 are associated with the downregulation of klotho, which may explain the increase in inflammatory factors during the aging 
process. Therefore, upregulation of klotho can suppress aging-related inflammation, slow down the aging process and extend life span.

To determine the mechanism of action of the klotho protein in COPD, BEAS-2B cells were treated with CSE, and the results showed that klotho expression decreased, while IL-6 and TNF- $\alpha$ expressions increased, relative to normal pulmonary epithelial cells. When the cells were transfected with klotho overexpression vector, and the control cells were transfected with blank vector before treatment with CSE, the expressions of IL-6 and TNF- $\alpha$ were significantly suppressed, while the expressions of klotho proteins were increased. These results demonstrate that klotho can prevent inflammation, and they are consistent with the results of previous studies $[4,5]$.

Recent studies [11,12] have also indicated that RIG-I is associated with inflammation. In various chronic inflammation-related diseases, the levels of expressions of RIG-I and pro-inflammatory factors are increased, and RIG-I expression is induced in senescent cells, leading to NF-KB activation. Inflammatory factors such as IL-6 and IL-8 are dependent on activated NF-KB and activated protein 1 (AP-1). Silencing RIG-I in aging cells significantly decreased the activation of NF-kB, which further supports the key effect of NF-KB on RIG-1-induced expressions of IL-6 and IL-8 $[13,14]$. A study has shown that the RIG-1induced activation of IL-6 and IL-8 was suppressed by klotho in a dose-dependent manner [5]. The combination of klotho and RIG-I suppressed RIG-I multimerization, and blocked its signal transduction, thereby increasing the expression levels of inflammatory factors. Thus, klotho works as an upstream gene that affects the anti-inflammation process.

The underlying pathophysiological mechanism of COPD involves airway and lung inflammation, while cigarette smoke is the main risk factor for its pathogenesis. The expressions of RIG-I, NF$\mathrm{KB}$, IL- 6 and TNF- $\alpha$ were increased in CSEtreated BEAS-2B cells, and were enhanced by inflammation. However, the expression level of klotho protein was decreased and negatively correlated with CSE such that CSE decreased klotho expression in a dose-dependent manner.

After transfecting cells with klotho overexpression vector, and transfecting control cells with blank vector prior to CSE exposure, RT-qPCR, Western blotting and ELISA were used to determine the mRNA and protein expression levels of RIG-I, NF-KB, IL-6 and TNF$\alpha$. The results showed that the expression levels of RIG-I and NF-kB decreased after upregulation in klotho protein expression in CSE-treated cells, demonstrating that klotho acted as an antiinflammatory upstream gene for RIG-I and NF$\mathrm{kB}$. However, the anti-inflammatory effect of klotho was weaker than that of dexamethasone. Based on previous findings, it can be hypothesized that klotho inhibits inflammation by regulating the RIG-I/NF-kB pathway.

The therapeutic aim for stable COPD is to reduce the frequency of acute events and increase the quality of life of patients. Most western therapy recommend the use of inhaled bronchial dilating agents and hormones, which can improve lung function and exercise tolerance. However, some patients do not favor these treatments due to high cost, difficulties associated with usage, and concern for adverse effects. In particular, patients in stable phase of the disease are unwilling to use these therapies for long because of occasional symptoms such as asthma and chest tightness.

Bufei yishen formula was developed by Professor Wang, a leading doctor in the Department of Chinese Medicine in Huaian Hospital of Chinese Traditional Medicine, Jiangsu Province. It is composed of six herbs: astragalus, ginseng, rehmannia, cornus, epimedium and atractylodes, and is effective in treating patients with stable COPD. Previous studies have shown that bufei yishen is safe and effectively alleviates symptoms, and improves lung function in patients with stable COPD [15]. Some domestic scholars have found that the six herbs in the formula not only promote lung and kidney invigoration but also prevent inflammation and aging [16-26]. As an anti-aging protein, klotho can prevent inflammation induced by COPD. Thus, it was hypothesized that the formula prevents inflammation and cures COPD by regulating klotho expression through the RIGI/NF-KB pathway.

To test this hypothesis, cytotoxicity analysis was first performed. Low doses of the formula were expected to promote BEAS-2B cell proliferation, while high doses were expected to suppress cell growth or even lead to cell death. Therefore, a 1:200 dilution which did not have a significant impact on cell proliferation, was chosen for subsequent experiments. Next, the effect of the formula on klotho expression was determined by treating BEAS-2B cells with the formula. Western blotting results showed that the formula promoted klotho protein expression. Then, further studies were carried out to verify whether the formula could prevent inflammation and slow down aging in the COPD cell model. BEAS-2B 
cells were treated with the formula for $24 \mathrm{~h}$ after CSE treatment, and inflammation-related pathways and biomarkers were determined.

Results from qPCR and Western blotting showed that, compared with the control group treated with CSE only, inflammation in the formulatreated group was significantly alleviated, while the mRNA and protein expression levels of RIG-I and NF-kB were decreased. Furthermore, ELISA results showed that the expression levels of IL-6 and TNF- $\alpha$ were significantly reduced, indicating that the formula can prevent inflammation, although its effect was weaker than that of the positive control dexamethasone). Thus, klotho protein expression suppressed the RIG-I/NF-KB inflammatory pathway, while Bufei yishen formula promoted klotho expression.

It was initially hypothesized that the formula prevented inflammation by upregulating klotho expression thereby suppressing the RIG-I/NF-KB inflammatory pathway. To verify the hypothesis, klotho expression was knocked down in BEAS2B cells and the klotho/RIG-I/NF-kB pathway induced by CSE treatment was blocked. The results showed that the anti-inflammatory effect of the formula became weaker with klotho knockdown, as indicated by the mRNA expressions of RIG-I and NF-KB and the protein expressions of RIG-I, NF-kB, IL-6 and TNF- $\alpha$. Therefore, based on these data, Bufei yishen formula can prevent inflammation and aging by upregulating klotho protein expression, thereby influencing the RIG-I/NF-kB pathway and downregulating IL-6 and TNF- $\alpha$ expressions.

\section{CONCLUSION}

To the best of our knowledge, this is the first study on the relationship between klotho and the RIG-I/NF-KB pathway in BEAS-2B cells in relation to the anti-inflammatory and anti-aging mechanisms of klotho. It is also the first study on the impact of Bufei yishen formula on the expressions of inflammatory factors and klotho in a COPD cell model. The results obtained further confirm that the formula prevents inflammation by upregulating klotho expression and downregulating IL-6 and TNF- $\alpha$ expressions via RIG-I/NF-kB pathway. These findings provide a strong basis for the clinical application of the formula.

\section{DECLARATIONS}

\section{Acknowledgement}

This study was supported by the Fifth Phase "333 Project" on Science Research of Jiangsu
Science and Technology Department (no. BRA2017248), the International Science \& Technology Cooperation Program of China (no. 2014DFA31940), the National Natural Science Foundation of China (nos. 81572259 and 81302011), and the Jiangsu Provincial Key Discipline of Medicine (no. ZDXKA2016003). The authors thank Qian Wang for guidance on study design and experiments, and Dingbo $\mathrm{Hu}$ for statistical analysis.

\section{Conflict of interest}

The authors have no conflict of interest to report with regard to this work.

\section{Contribution of authors}

We declare that this work was done by the author(s) named in this article and all liabilities pertaining to claims relating to the content of this article will be borne by the authors. All authors read and approved the manuscript for publication. Jian-qing $\mathrm{Wu}$ conceived and designed the study, Wei-min Wang, Long-chuan Wu, Bo Chen, Yan Li, Yu Chen, Xing-xing Xu, Jian-qing $\mathrm{Wu}$ collected and analysed the data, Wei-min Wang wrote the manuscript.

\section{REFERENCES}

1. Vogelmeier CF, Criner GJ, Martinez FJ, Anzueto A, Barnes PJ, Bourbeau J, Celli BR, Chen R, Decramer M, Fabbri LM, et al. Global Strategy for the Diagnosis, Management, and Prevention of Chronic Obstructive Lung Disease 2017 Report. GOLD Executive Summary. Am J Respir Crit Care Med 2017; 195(5): 557-582.

2. Rahman I, Adcock IM. Oxidative stress and redox regulation of lung inflammation in COPD. Eur Respir $J$ 2006; 28(1): 219-242.

3. Rahman I, van Schadewijk AA, Crowther AJ, Hiemstra PS, Stolk J, MacNee W, De Boer WI. 4-Hydroxy-2nonenal, a specific lipid peroxidation product, is elevated in lungs of patients with chronic obstructive pulmonary disease. Am J Respir Crit Care Med 2002; 166(4): 490495.

4. Kurosu H, Yamamoto M, Clark JD, Pastor JV, Nandi A, Gurnani P, McGuinness OP, Chikuda H, Yamaguchi $M$, Kawaguchi $\mathrm{H}$, et al. Suppression of aging in mice by the hormone Klotho. Sci 2005; 309(5742): 1829-1833.

5. Maekawa Y, Ishikawa K, Yasuda O, Oguro R, Hanasaki H, Kida I, Takemura Y, Ohishi M, Katsuya T, Rakugi $H$. Klotho suppresses TNF-induced expression of adhesion molecules in the endothelium and attenuates NF-kB activation. Endocrine 2009; 35(3): 341-346.

6. Liu F, Wu S, Ren H, Gu J. Klotho suppresses RIG-Imediated senescence-associated inflammation. Nat Cell Biol 2011; 13(3): 254-262.

Trop J Pharm Res, October 2018; 17(10): 1930 
7. Li H, Wang WM, Liu YY, Wang ZF. Impact of Bufei Yishen Formula on lung function and life standard in COPD stable period. J Sichuan Chin Med 2015; 1(33): 86-88.

8. World Health Organization. Declaration of Helsinki. Br Med J 1996; 313(7070): 1448-1449.

9. Kuilman T, Michaloglou C, Vredeveld LCW, Douma S, van Doom R, Desmet CJ, Aarden LA, Mooi WJ, Peeper DS. Oncogene-induced senescence relayed by an interleukin-dependent inflammatory network. Cell 2008; 133(6): 1019-1031.

10. Acosta JC, O'Loghlen A, Banito A, Guijarro MV, Augert A, Raguz S, Fumagalli M, Da Costa M, Brown C, Popov N, et al. Chemokine signaling via the CXCR2 receptor reinforces Senescence. Cell 2008; 133(6): 1006-1018.

11. Wang J, Wu S, Jin X, Li M, Chen S, Teeling JL, Perry $\mathrm{VH}, \mathrm{Gu} J$. Retinoic acid-inducible gene-l mediates late phase induction of TNF by lipopolysaccharide. J Immunol 2008; 180(12): 8011-8019.

12. Poeck H, Bscheider M, Gross O, Finger K, Roth S, Rebsamen $M$, Hannesschlager $N$, Schlee $M$, Rothenfusser $S$, Barchet $W$, et al. Recognition of RNA virus by RIG-I results in activation of CARD9 and inflammasome signaling for interleukin $1 \beta$ production. Nat Immunol 2010; 11(1): 63-69.

13. Yoneyama $M$, Kikuchi $M$, Natsukawa $T$, Shinobu $N$, Imaizumi T, Miyagishi M, Taira K, Akira S, Fujita T. The RNA helicase RIG-I has an essential function in doublestranded RNA-induced innate antiviral responses. Nat Immunol 2004; 5(7): 730-737.

14. Kato H, Sato S, Yoneyama M, Yamamoto M, Uematsu S, Matsui K, Tsujimura T, Takeda K, Fujita T, Takeuchi O, et al. Cell type-specific involvement of RIG-I in antiviral response. Immun 2005; 23: 19-28.

15. Blake DJ, Reese CM, Garcia M, Dahlmann EA, Dean A. Soluble extracellular Klotho decreases sensitivity to cigarette smoke induced cell death in human lung epithelial cells. Toxicol In Vitro 2015; 29(7): 1647-1652.

16. Yao LP, Guang LS, Li NL. Research on IL-6 and IFN in viral myocarditis patients treated by astragalus membranaceus. Chin J Postgraduates Med 2005; 28(7): 41-42.

17. Zhou YJ, Lu S, Wang ZY. Effect of anti-inflammation and anti-oxidant on COPD model mice injected with astragalus membranaceus in enterocoelia. Jinagsu Med J 2007; 33(7): 709-711.

18. Zhang $W$, Zhao $Y M$, Zhang $X Y$. Intervened effect of Renshen Gejie San on $k B$ andy-GCS expressions in COPD model mice. J Shandong Univ Tradit Chin Med 2006; 3(5): 399-401.

19. Zhou Y, Yang B, Jiang $R$, Yao $X$, Wang YP. Aging postponement and related mechanisms of ginsenoside on hemopoietic stem cells. Nat Med J China 2010; 90(48): 3421-3425.

20. Zhao X, Li P, Sun Y. HPLC/ESI-MS analysis of the Radix Rehmanniae Praeparata. Chin J Pharm Anal 2007; 27(3): 874-876.

21. Xia $Q H, L U Q$ L. Pharmacological progress on radix rehmanniae praeparata. J Jiangxi Univ Tradit Chin Med 2008; 20(6): 96-97.

22. Ou $Q$, Ge TD, Wang $D D$, Wang $Y X$. Relation between anti-aging HDF and cyclinD1 expression in polysaccharide. Heilongjiang Med \& Pharm 2008; 31(1): 1-3.

23. Cheng $Z Q$, Miao $L Y$, Jun $X U$, Luo J. Study of the effect of Zidianling on anti-inflammatory hemostasia and immunosuppression in mice. Chin J Hosp Pharm 2007; 27(11): 1512-1514.

24. Dong JC, Shi ZY, Shen ZY, Wang WJ, Li YL, Zhang QF, Jin WY. Clinical study of inhalation of large dosage of corticosteroid and kidney-tonifying herbs in treatment of steroids dependent asthmatic patients. Chin J Integr Tradit West Med 1994; 14(8): 458-461.

25. Xie JY, Dong JC, Gong ZH, Zhao FD, Cui Y. Impact of herba epimedii and astragalus membranaceus on mice TNF- $\alpha$ and NF-KB. Chin J Integr Tradit West Med 2006; 26(8): 723-726.

26. Wu LF, Yuan YB, Wang KF, Li SQ, Liu J. Research progress on chemistry and pharmacology of hydrolyzable tannin monomer. Chin Tradit Herb Drugs 2014; 45(2): 290-299. 